\title{
PROFILE: HIV IN NORTH WEST PROVINCE, SOUTH AFRICA
}

\author{
E Variava \\ Specialist Physician, Head of Internal Medicine and Wellness Unit, Klerksdorp-Tshepong Complex, North West
}

North West Province (NWP) has one of the highest HIV prevalences in South Africa. It is further challenged by severe poverty, a huge surface area housing a scattered community, and limited human resources. Despite this, it is one of the provinces that have successfully initiated large-scale ARV access in South Africa. This article describes the challenges and solutions that the province has grappled with to improve access to care for people with HIV.

Matlosana is a district of the Southern Region of the NWP and was previously referred to as KOSH (Klerksdorp, Orkney, Stilfontein and Hartebeesfontein). The Southern and Bojonala regions have the highest HIV ANC prevalence rates in the province $(31.1 \%$ and $30.4 \%$ respectively, the provincial rate being 26.7\%) (Fig. 1).

Possible reasons for these figures include a highly mobile population:

Klerksdorp is located along the N12, a major truck route to the Northern Cape, Namibia and Botswana.

- Klerksdorp is a major trading post for surrounding areas that lack services such as retail, health, academic and training institutions.

- There is a high proportion of migrant workers (goldminers who are often not unionised contract workers and hence have no mine medical cover).

Klerksdorp-Tshepong Hospital Complex (KTC) resembles most secondary-level hospitals in South Africa in that it provides primary hospital care for those living in the immediate vicinity (Matlosana), secondary care for patients from all primary care hospitals in the Southern and Bophirima regions, limited tertiary services for the entire NWP (renal unit, MDR TB unit, oncology unit, burns unit) and on occasion quaternary care (highly infectious unit for outbreaks of viral haemorrhagic fevers such as Congo fever).

Large numbers of public secondary-level institutions are seeing increased numbers of patients, have severe budgetary constraints, face shortages of skilled personnel, particularly senior clinical and auxiliary staff (senior medical officers, general specialists, psychologists, social workers, senior nurses, senior pharmacists, etc.) and lack diagnostic and therapeutic tools. Junior staff, mainly interns and community service doctors, are often called upon to provide a wide range of services as these institutions are inundated with complicated and difficult cases referred from their primary referral sites.
The rollout of the South African government's Plan for Comprehensive Care for HIVIAIDS in the Public Health Sector has created new challenges that have shaken the practice of medicine. An old cliché that not only defines health as a physical state of wellness but also includes social, psychological and economic sufficiency can best be realised by the goal of the Comprehensive Care package. 'HIV medicine' is no longer a clinical entity. It forces us to consider other pertinent issues in relation to the growing pandemic, which include a study of interpersonal relations, sexuality in diverse cultures, death and dying and being culturally sensitive, the right to and access to disability/support grants, early identification of families in dire need, orphans and their support, food security, home-based care availability, and a host of other no less important local issues (informal settlements, water, sanitation, etc.).

Not only do 'HIV clinicians' have to deal with 'non-medical' issues, HIV as a disease also confounds the most astute of pure clinicians because of its multi-system manifestations. These include autoimmune phenomena (idiopathic thrombocytopenic purpura, thrombotic thrombocytopenic purpura, haemolysis, etc.), opportunistic infections (TB, Mycobacterium avium complex, cryptococcal meningitis, pneumocystis pneumonia, etc.), malignancy (Kaposi's sarcoma, lymphoma, etc.) and antiretroviral therapy (ART) with its complications. Furthermore HIV can be a coincidental finding in patients with other diseases (hypertension, diabetes, cardiac, pulmonary, renal, etc.), often leading to diagnostic and therapeutic dilemmas. Often extensive (expensive, difficult and timeconsuming) investigations and procedures are undertaken to diagnose a patient's presenting condition. These range from radiological (X-rays, ultrasound, computed tomography scans) and laboratory (histology for biopsies, cultures of material/specimens, serology, etc.) to invasive procedures (bronchoscopy, endoscopy). Doctors who work in high HIV prevalence areas become familiar with HIV-associated illnesses that were previously rare or uncommon (PCP/Crypto), or common diseases (such as extrapulmonary tuberculosis or 


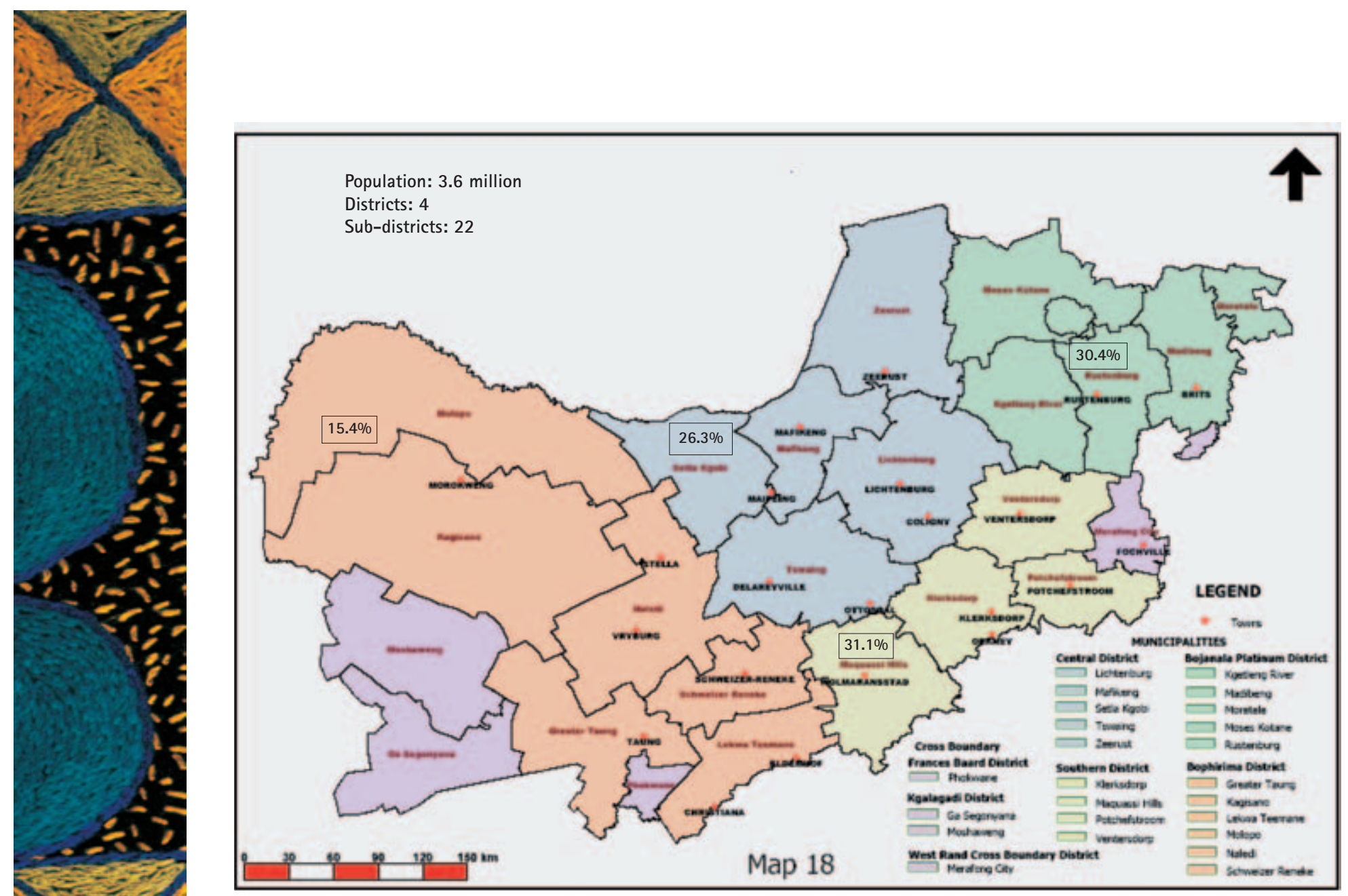

Fig. 1. North West Province: district and local municipalities (source: Municipal Demarcation Board, 2002).

pneumonias) presenting differently. Sub-specialist support for diagnosis and therapy is lacking at most secondary hospitals, and this further frustrates the clinician.

With increasing access to antiretrovirals (ARVs), a more endemic/chronic form of HIV now coexists leading to newer disease entities including multidrug-resistant or mutant HIV, lactic acidosis, lipoatrophy, lipodystrophy, metabolic syndromes, reconstitution disease (tuberculosis, crypto, hepatitis, MAl, etc.) and other toxicities induced by ART.

Clinicians working in areas where the prevalence of HIV is high, specialist support is scarce and ARV rollout is expanding require ongoing training, as a patient can incidentally be found to be HIV positive (asymptomatic or on therapy) and present with a non-HIV-related ailment or with various 'sub-specialist complaints' (pericardial TB, spinal TB, renal failure, etc.) directly attributable to HIV or its related opportunistic diseases or effects of therapy.

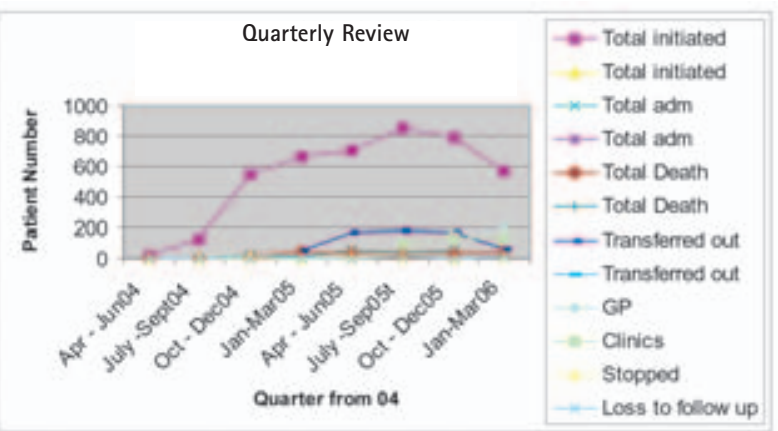

Fig. 2. Quarterly review, April 2004 - March 2006.
Tshepong Hospital is located in a high HIV prevalence region (31.1\%) (NWP ANC seroprevalence study, 2004) and the Department of Medicine deals with large numbers of acutely ill HIV-infected individuals (accounting for 60\% of admissions). An average of 250 new cases of HIV are diagnosed each month (local hospital statistics), many present with advanced (stage III/IV) disease and CD4 counts of $<200 / \mu$, and many are malnourished. Our crude fatality rate averages 16\% (3 - 5 deaths a day), and HIV-related disease was found to be a major contributor. An audit presented at the 2005 AIDS Congress in Durban showed that $45 \%$ of deaths were confirmed as HIV positive, another 20\% were clinically positive, in 30\% HIV status was unknown, and only 5\% were confirmed HIV negative (mortality analysis in the Department of Medicine, Klerksdorp/Tshepong Hospital Complex, July 2003 - June 2004).

The Department of Medicine is responsible for 160 acute medical beds and 80 step-down beds with a bed occupancy rate of $80 \%$ and an average length of stay of 8 days. The stepdown unit provides palliative, recuperative and rehabilitative care. While the Department of Health in NWP prepared its rollout plan in October 2003, KTC began setting up a Wellness Unit. With help from local business, renovations to the site were undertaken and the site was officially opened by our previous MEC for Health (Dr Sefularo). Partnerships with NGOs including Hospice, Lifeline and Seboka facilitated recruitment and training of lay counsellors. Faith-based organisations assisted with provision of food security for the destitute. Provincial support for a local HIV seminar in 2003 provided the original impetus for the training of doctors and nurses. RHRU 
TABLE I. SUMMARY OF QUARTERLY STATS FOR THE WELLNESS UNIT, PRESENTED AT KTC QUARTERLY REVIEWS

\begin{tabular}{|c|c|c|c|c|c|c|c|c|c|}
\hline & $\begin{array}{l}\text { Apr - } \\
\text { Jun } 04\end{array}$ & $\begin{array}{l}\text { July - } \\
\text { Sept } 04\end{array}$ & $\begin{array}{c}\text { Oct - } \\
\text { Dec } 04\end{array}$ & $\begin{array}{c}\text { Jan- } \\
\text { Mar } 05\end{array}$ & $\begin{array}{l}\text { Apr - } \\
\text { Jun } 05\end{array}$ & $\begin{array}{l}\text { July - } \\
\text { Sep } 05\end{array}$ & $\begin{array}{c}\text { Oct - } \\
\text { Dec } 05\end{array}$ & $\begin{array}{c}\text { Jan - } \\
\text { Mar } 06\end{array}$ & Total \\
\hline Total initiated & 25 & 124 & 548 & 667 & 704 & 851 & 785 & 566 & 4270 \\
\hline Total adm. & 0 & 4 & 26 & 6 & 50 & 47 & 50 & & 183 \\
\hline Total deaths & 4 & 2 & 14 & 41 & 31 & 23 & 27 & 40 & 182 \\
\hline Transferred out & & & & 48 & 168 & 183 & 168 & 67 & 634 \\
\hline GP & & & & & & & 110 & 214 & 324 \\
\hline Clinics & & & & & & 100 & 138 & 127 & 365 \\
\hline Stopped ART & 0 & 0 & 6 & 3 & 6 & 9 & 5 & 8 & 37 \\
\hline Lost to follow-up & 0 & 0 & 1 & 0 & 2 & 13 & 0 & 6 & 22 \\
\hline Total follow-up & & & & & & & & & 2706 \\
\hline
\end{tabular}

and later AURUM consolidated the training. Partnering with our district primary care team enabled us to develop staging sites at local clinic level and strengthened the referral process.

Provincial will and support led to the Wellness Unit with its multidisciplinary team being fully established at the point of official accreditation in July 2004.

In line with the Provincial Plan, KTC assisted other sites with their programme implementation by assisting with staff training, accreditation and treatment of patients from these sites. Taung, Vryburg, Ganyesa and Potch have all been accredited and patients from these areas were successfully referred back.

As the programme is rapidly growing (initiating ARVs in an average of 240 patients per month) (Wellness Unit stats, KTC quarterly reviews - Table I) the need to refer stable patients (with good immunological and clinical response) to their local clinics has become more apparent. Clinics that were originally staging sites are now seeing and distributing medication for the down-referred patients (pre-packed for each patient and delivered to the clinic by the hospital pharmacy). A pilot programme for down-referral is referring stable patients to a local GP network (funded by Broadreach) where the GPs receive a capitation fee for following up these patients. Downreferred patients return to hospital 6-monthly for ongoing tests. As a result more space at the Wellness Centre has been created, enabling us to continue with initiating newer patients on ARVs. There are also cases in which earlier initiation or hospital-based initiation of ARVs needs to be considered. The logical next phase would be the initiation of ARVs in stable patients at the PHC level. Plans are being developed and it is hoped that by 2007 selected clinics may begin initiating ARVs provided they meet requirements for accreditation.

The complexities of HIV and a rapidly expanding HIV programme mean a growing need to form strategic partnerships. With this in mind we at KTC have pursued partnerships with RHRU, AURUM, Broadreach, Hospice, Lifeline, Seboka, primary care clinics, the local GP network and other care providers in Matlosana. The formation of the HIV Clinicians Society has given us a means of disseminating medical information to caregivers in our areas.

Thanks to the persistent Tanya Nielson, an enthusiastic pharmacist who worked hard in setting up our branch, our inaugural launch (attended by 100 care providers) was a success. Attendees included doctors, pharmacists, dieticians, primary care nurses, social workers and home-based care groups, all part of a multidisciplinary team needing to understand HIV from authorities in their field. Dr Francois Venter presented an overview of the rollout programme and the challenges posed by ARV provision. Professor Churchyard presented a talk on TB and HIV and how ARV will impact on the TB epidemic.

A second meeting was held in May and again this was enthusiastically supported, encouraging us to continue.

The North West Provincial Department of Health, KTC management, and the staff of the Wellness Unit all need to be thanked for the success of the unit. 